\title{
Plasma DNA level in predicting therapeutic efficacy in advanced nonsmall cell lung
} \section{cancer}

\author{
S. Kumar*, R. Guleria*, V. Singh*, A.C. Bharti ${ }^{*}$, A. Mohan* and B.C. Das
}

ABSTRACT: Assessment of total plasma DNA can be a promising noninvasive tool for monitoring the effect of cytotoxic therapies in order to predict therapeutic efficacy at an early stage.

Cell-free plasma DNA levels were quantified before the first, second and third cycle of chemotherapy in $\mathbf{4 2}$ patients with advanced nonsmall cell lung cancer and correlated with response to therapy, as assessed by computed tomography following the third chemotherapy cycle.

A significantly lower plasma DNA level, measured before various treatment cycles, was found in patients with remission or stable disease than in those with progression. Higher levels and insufficient decrease in plasma DNA levels during the course of chemotherapy indicated poor outcome. For predicting insufficient therapy response, a sensitivity of $26.9 \%$ was achieved at $100 \%$ specificity using plasma DNA levels before the first therapy cycle. Prediction of disease progression was achieved with a sensitivity of $35.7 \%$ at $100 \%$ specificity using plasma DNA levels before the first therapy cycle.

Monitoring of plasma DNA levels during the course of chemotherapy could identify patients who are likely to exhibit an insufficient therapeutic response and disease progression at an early stage. This may help in individualising treatment, and could lead to better management of advanced-stage lung cancer.

KEYWORDS: Biomarker, chemotherapy, response, sensitivity, specificity

ung cancer is the leading cause of cancerrelated death in India and across the globe [1]. Although the low 5-yr survival rate $(<15 \%)$ has changed minimally since the mid1980s, new agents are being identified in order to improve results. For the treatment of advancedstage lung cancer patients, in addition to first-line chemotherapy, second- and third-line chemotherapy and targeted therapeutic agents are a few of the treatment options currently in use [2-4]. However, lack of a simple and effective tool for predicting therapeutic efficacy early during the course of treatment has restricted progress in improving the survival of these patients. Although positron emission tomography, alone or in combination with computed tomography (CT), has shown some potential in the estimation of therapeutic efficacy [5-7], the low sensitivity, detection of only macroscopic alterations, and that after several cycles of cytotoxic therapy, and the risk of radiation exposure has limited their utility for monitoring therapeutic efficacy at an early stage. Thus there is a growing need for more effective diagnostic tools for: 1) prediction of the prognosis of the patient, 2) monitoring of the treatment course, and 3) detection of response to therapy at an early stage. This would help in optimising disease management on an individual basis. Biomarkers present in the circulation are promising candidates since their kinetics can reflect the effect of cytotoxic therapies. The advantage associated with biomarkers is that they take into account the heterogeneity as well as the activity of the tumour mass. Further, serial assessment of biomarkers during cytotoxic therapy may help in early prediction of therapeutic efficacy.

The finding that tumours are capable of shedding DNA into the blood stream, which can be recovered from both serum and plasma and used as surrogate source of tumour DNA, has opened new areas of diagnosis and prognosis [8]. Apoptotic cell death is known to be the major source of cell-free DNA present in the circulation (cfDNA). It is noteworthy that most cytotoxic therapies cause cell death by apoptosis, leading to the release of cfDNA. As the quantification of

\section{AFFILIATIONS}

*Dept of Medicine, All India Institute of Medical Sciences, New Delhi, "Division of Molecular Oncology, Institute of Cytology and Preventive Oncology, Noida, and

"Dr. B. R. Ambedkar Centre for Biomedical Research, University of Delhi, Delhi, India.

\section{CORRESPONDENCE}

R. Guleria

4083 A, Teaching Block-4 $4^{\text {th }}$ Floor

Dept of Medicine

All India Institute of Medical

Sciences

Ansari Nagar

New Delhi-110029

India

E-mail: randeepg@hotmail.com

Received:

Nov 252009

Accepted after revision:

Feb 052010

First published online:

Feb 252010 
the cell death in tumour tissues normally requires invasive procedures and assesses only part of the heterogeneous pathophysiological processes, analysis of the kinetics of cfDNA may be an alternative for monitoring of cytotoxic therapy as it can easily be measured serially during the course of the disease and treatment.

Keeping these observations in focus, the present study was designed with the aim of analysing the efficacy of levels of cfDNA for monitoring first-line chemotherapy in a homogeneous group of patients with advanced nonsmall cell lung cancer (NSCLC). The primary objective was to determine whether or not cfDNA levels can predict response to therapy or disease progression $(\mathrm{P})$ at an early stage in advanced NSCLC. Additionally, the utility of cfDNA as a prognostic factor for survival in NSCLC patients was assessed.

\section{MATERIALS AND METHODS}

\section{Patients}

In this prospectively performed study, 42 newly diagnosed and untreated advanced-stage NSCLC patients were evaluated. Only those patients who received a minimum of three cycles of chemotherapy were included in the study. A total of 100 controls (patients with benign pulmonary diseases, including 32 with chronic obstructive pulmonary disease, 26 pulmonary tuberculosis, 33 sarcoidosis, four bronchiectasis and five interstitial lung disease) were also included. All patients were enrolled from the outpatient Dept of Medicine of the All India Institute of Medical Sciences (New Delhi, India) during the years 2006-2009. For all patients, a diagnosis of lung cancer was confirmed by histological examination of biopsy and/or cytology specimens obtained during fibreoptic bronchoscopy or with a CT-guided procedure. Pretreatment assessment included evaluation of Eastern Cooperative Oncology Group (ECOG) performance status, radiography and CT scan of the chest and upper abdomen. If necessary, a CT or magnetic resonance imaging scan of the brain and radionuclide bone scan were performed. All of the patients were staged according to the recommendations made by the International Association for the Study of Lung Cancer (IASLC) Staging Committee for NSCLC [9]. Epidemiological data, including demographics, stage, tumour size and histopathological data, and risk factors, including smoking habit and alcohol intake, were also recorded. A smoker was defined as a person who smoked $>20$ pack-yrs bidis or cigarettes during their lifetime (a bidi being a traditional Indian smoking product that is made with aged Indian tobacco, hand wrapped in a tembhurni leaf, nubbed on one end and bound with a thin string on the other). A never-smoker was defined as a person who had not smoked $>100$ bidis/cigarettes in their lifetime. Smoking in pack-years was calculated as the product of smoking duration (in years) and the mean number of packs (i.e. 20 bidi/cigarette equivalents) smoked daily. Alcohol habit was defined based on a previous report [10]. Alcohol drinkers were defined as people who drink alcoholic beverages at least once daily for $\geqslant 6$ months. The quantity of alcohol drunk daily was the product of the amount consumed (in millilitres) and the percentage of alcohol in the product (assuming 3\% for beer, $10 \%$ for toddy and $40 \%$ for the remaining alcoholic drinks). The quantity of Indian and non-Indian alcohol consumed was the sum of the alcohol amount from the relevant beverages, as specified above. In patients with NSCLC, response to therapy was classified according to the World Health Organization (WHO) guidelines, defining complete remission (CR) as compete disappearance of all tumour lesions, partial remission (PR) as tumour reduction by $\geqslant 50 \%, P$ as tumour increase by $\geqslant 25 \%$ or the appearance of new tumour manifestations, and stable disease (SD) as tumour reduction by $<50 \%$ or increase by $<25 \%$ [11]. The radiology reviews performed for response measurements were independent and blinded. The study was approved by the Ethics Committee of the All India Institute of Medical Sciences, and written informed consent was obtained from all of the patients.

\section{Analysis of cfDNA levels}

Venous blood was collected in sterile EDTA-coated vials from all subjects at baseline (for determination of baseline cfDNA level before first chemotherapy cycle (BV1)). Blood was also collected before the second (for BV2) and third cycles of chemotherapy (for BV3). Within $1 \mathrm{~h}$, samples were centrifuged $(2,500 \times g$ for $10 \mathrm{~min}$ ) and the plasma was removed, aliquoted and stored at $80^{\circ} \mathrm{C}$ until further analysis. DNA from stored plasma fractions was isolated using the QIAamp DNA Blood Mini Kit (QIAGEN, Valencia, CA, USA) as per the manufacturer's instructions. The time from plasma separation and storage to performing plasma DNA extraction and quantification was $<6$ months. In addition, in order to avoid any variation in plasma DNA levels, the storage time and temperature were kept comparable for all samples. The isolated DNA was stored at $-20^{\circ} \mathrm{C}$ until further use. cfDNA was quantified using the PicoGreen dsDNA Kit (Molecular Probes, Eugene, OR, USA) according to the manufacturer's instructions. PicoGreen reagent is an ultra-sensitive fluorescent nucleic acid stain for the quantification of double-stranded DNA in solution. Briefly, PicoGreen dye was diluted 1:200 with TE buffer (10 mM Tris-HCl, 1 mM EDTA; pH 7.5). Each reaction contained $50 \mu \mathrm{L}$ diluted PicoGreen dye solution plus a sample of DNA made up to $50 \mu \mathrm{L}$ in TE buffer. Each DNA sample was analysed in two duplicated dilution series. An ELISA plate containing these reagents was read in the Synergy HT Multi-Mode microplate reader (Bio-Tek, Winooski, VT, USA) at an emission wavelength of $520 \mathrm{~nm}$ and excitation wavelength of $480 \mathrm{~nm}$. In order to minimise photobleaching effects, the time for fluorescence measurement was kept constant for all samples. Blank values were subtracted and replicates averaged for each sample. Standard curves were constructed by serial dilution of the lambda DNA stock provided by the manufacturer. Plasma DNA concentrations were determined from a standard curve. The people performing the plasma DNA quantification were blinded to the clinical outcome of the patients at the time of testing.

In the present study, cfDNA levels were compared in patients with $\mathrm{P}$ and those showing remission (R; $\mathrm{CR}+\mathrm{PR})$ and $\mathrm{SD}$ in order to predict $\mathrm{P}$ (evaluation 1). In addition, cfDNA levels in patients with $\mathrm{R}$ were compared with those exhibiting $\mathrm{P}$ and SD in an effort to predict insufficient response to therapy (evaluation 2).

\section{Statistical analysis}

In order to test the association of response to therapy with the overall survival of the patients, Kaplan-Meier curves and logrank analyses were established for various response groups. 
Concerning the biochemical variables, BV1, BV2 and BV3, and the percentage changes between them (BV1-BV2 and BV1-BV3), were considered for statistical analyses. The Kruskal-Wallis test was applied to measure the significance of cfDNA level in predicting response to therapy. If found significant, a post-hoc analysis was performed to compare their levels between any two response groups (Bonferroni correction applied; $\mathrm{p}<0.017$ was taken as a significant value) by Wilcoxon rank-sum test. Friedman's test was applied for comparing successive cfDNA levels within a group. Multiple comparisons were performed using the post-hoc Wilcoxon signed-rank test. Further, cfDNA level was evaluated for its power to univariately discriminate between: 1$)$ patients with $\mathrm{P}$ and without $\mathrm{P}(\mathrm{R}+\mathrm{SD}$; evaluation 1$)$,

\begin{tabular}{|c|c|c|c|}
\hline & Patients & Survival days & p-value \\
\hline \multicolumn{4}{|l|}{ Age } \\
\hline$<60$ yrs & $27(64.3)$ & $370.0(297.0-443.0)$ & 0.346 \\
\hline$\geqslant 60$ yrs & $15(35.7)$ & 328.0 (317.4-338.6) & \\
\hline \multicolumn{4}{|l|}{ Stage } \\
\hline $111^{\circ}$ & $23(54.8)$ & $416.0(364.3-467.7)$ & 0.149 \\
\hline IV & $19(45.2)$ & $324.0(290.5-357.5)$ & \\
\hline \multicolumn{4}{|l|}{ ECOG grade } \\
\hline 1 & $30(71.4)$ & $393.0(266.4-519.6)$ & 0.275 \\
\hline $2-3$ & $12(28.6)$ & $333.0(307.3-358.7)$ & \\
\hline \multicolumn{4}{|l|}{ Histology } \\
\hline Squamous cell cancer & $31(73.8)$ & $367.0(280.1-453.9)$ & 0.620 \\
\hline Adenocarcinoma & $11(26.2)$ & $370.0(309.3-430.7)$ & \\
\hline \multicolumn{4}{|l|}{ Smoking status } \\
\hline Smoker & $36(85.7)$ & $370.0(301.4-438.6)$ & 0.495 \\
\hline Never-smoker & $06(14.3)$ & 324.0 (248.9-399.1) & \\
\hline \multicolumn{4}{|l|}{ Alcohol habit } \\
\hline Yes & 25 (59.5) & $370.0(300.3-439.7)$ & 0.499 \\
\hline No & $17(40.5)$ & $348.0(307.4-388.6)$ & \\
\hline \multicolumn{4}{|l|}{$T$ factor } \\
\hline Т2-Т3 & $17(40.5)$ & $370.0(246.3-493.7)$ & 0.057 \\
\hline $\mathrm{T} 4$ & 25 (59.5) & $332.0(276.3-387.7)$ & \\
\hline \multicolumn{4}{|l|}{$\mathrm{N}$ factor } \\
\hline No-N1 & $21(50.0)$ & $394.0(275.2-512.8)$ & 0.278 \\
\hline N2-N3 & $21(50.0)$ & $333.0(304.8-361.2)$ & \\
\hline \multicolumn{4}{|l|}{ Tumour location } \\
\hline Right & $23(54.8)$ & $394.0(354.5-433.5)$ & $0.036^{+}$ \\
\hline Left & $19(45.2)$ & $322.0(283.5-360.4)$ & \\
\hline \multicolumn{4}{|l|}{ Pleural effusion } \\
\hline Yes & $13(31.0)$ & $328.0(285.0-371.0)$ & 0.309 \\
\hline No & $29(69.0)$ & $370.0(295.4-444.6)$ & \\
\hline \multicolumn{4}{|l|}{ Chemotherapy regimen } \\
\hline Paclitaxel+carboplatin & $20(47.6)$ & $394.0(350.7-437.3)$ & $0.166^{\S}$ \\
\hline Cisplatin+etoposide & $20(47.6)$ & $328.0(308.8-347.1)$ & \\
\hline Gemcitabine+carboplatin & $2(4.8)$ & 333.0 & \\
\hline
\end{tabular}

Data are presented as $\mathrm{n}(\%)$ or median (95\% confidence interval), unless otherwise stated. ECOG: Eastern Cooperative Oncology Group (performance status); T: tumour; N: node. ${ }^{\#}: n=42 ;{ }^{~}:$ stages IIIA ( $\left.n=6\right)$ and IIIB $(n=17)$; ${ }^{+}$: significant observation; ${ }^{\text {s. }} \mathrm{p}$-value for survival difference in patients receiving paclitaxel plus carboplatin versus cisplatin plus etoposide. and 2) patients with $\mathrm{R}$ and without $\mathrm{R}(\mathrm{P}+\mathrm{SD}$; evaluation 2$)$ by Wilcoxon rank-sum test. In order to identify the diagnostic biomarker for insufficient therapeutic efficacy and $\mathrm{P}$, receiver operating characteristic (ROC) curves and the corresponding areas under the curve (AUCs) were calculated. In addition, sensitivity, positive predictive value, negative predictive value and cut-off values for detecting insufficient therapeutic efficacy and $\mathrm{P}$ were calculated at $100 \%$ specificity (with $95 \%$ confidence interval (CI)). In order to test the association of BV1, BV2 and BV3 with the overall survival of the patients, Kaplan-Meier curves and log-rank analyses were established using their median values as cut-off points. Total plasma DNA levels were compared between patients with NSCLC and controls using an independent-sample unpaired t-test. A p-value of $<0.05$ was considered significant. All statistical analyses were performed with the SPSS software program for Windows (SPSS 9.1; Stata Corporation, College Station, TX, USA).

\section{RESULTS}

\section{Patient's characteristics}

The median age of the 42 patients with NSCLC was 55.5 yrs (range 33-78 yrs), and $56.5 \mathrm{yrs}$ (range 35-86 yrs) in the 100 controls. The mean \pm SD plasma DNA levels were 95.1土 $32.1 \mathrm{ng} \cdot \mathrm{mL}^{-1}$ in NSCLC patients and $74.0 \pm 19.9 \mathrm{ng} \cdot \mathrm{mL}^{-1}$ in controls, the difference being highly significant $(p<0.001)$. The baseline characteristics of the patients with NSCLC are given in table 1. Briefly, 23 patients had stage III disease and 19 stage IV disease. The majority of the patients were smokers $(85.7 \%)$ and had squamous cell carcinoma (73.8\%). Of the patients, 20 each received paclitaxel plus carboplatin or cisplatin plus etoposide combination chemotherapy, whereas two received gemcitabine plus carboplatin for a minimum of three cycles.

\section{Correlation of patient characteristics with survival}

Staging investigations following the third cycle of chemotherapy showed that four (9.5\%) of the 42 NSCLC patients showed CR, 12 (28.6\%) PR, 14 (33.3\%) P and 12 (28.6\%) SD. Patients with PR were combined with those with $\mathrm{CR}$ to form the $\mathrm{R}$ group (16 out of $42 ; 38.1 \%$ ). The median follow-up time was 332.5 days (range 173-864 days). The median survival time was 367 days (95\% CI 310.0-424.0 days). Kaplan-Meier curves for overall survival showed highly significant differences in survival between patients with $\mathrm{R}$ and SD or $\mathrm{P}(\mathrm{p}<0.001)$, with median survival times of 528 (95\% CI 430.7-625.3), 327 (95\% CI 320.2-333.8) and 322 (95\% CI 298.3-345.6) days, respectively. For evaluation 1, in which patients with $\mathrm{R}$ and SD were compared with those with $\mathrm{P}$, a significant difference in overall survival was observed, with median survival times of 416 (95\% CI 356.5-475.5) and 322 (95\% CI 298.3-345.6) days, respectively $(\mathrm{p}<0.001)$. Similarly, patients who achieved $\mathrm{R}$ showed greater survival duration than those with $\mathrm{P}$ and SD (evaluation 2), with median survival times of 528 (95\% CI 430.7-625.3) and 327 (95\% CI 319.3-334.7) days, respectively $(\mathrm{p}<0.001)$.

The univariate survival analysis was also examined for variables, including age, stage, tumour factor, nodal status, performance status, histology, smoking and tobacco habits, and chemotherapy regimen (table 1). A significant trend for tumour location $(p=0.036)$ was observed. A nonsignificant trend for tumour size $(p=0.057)$ was also found. However, no 


\begin{tabular}{|c|c|c|c|c|c|c|c|c|c|}
\hline & \multirow[t]{3}{*}{$\mathbf{R}$} & \multirow[t]{3}{*}{ SD } & \multirow[t]{3}{*}{$\mathbf{P}$} & \multicolumn{6}{|c|}{ p-value } \\
\hline & & & & \multirow[t]{2}{*}{ Overall $^{\#}$} & \multicolumn{3}{|c|}{ Post-hoc analysis" } & \multicolumn{2}{|c|}{ Evaluation $^{+}$} \\
\hline & & & & & $R$ versus SD & $R$ versus $P$ & SD versus $P$ & $\begin{array}{c}\mathrm{R}+\mathrm{SD} \\
\text { versus } \mathrm{P}\end{array}$ & $\begin{array}{c}R \text { versus } \\
\text { SD+P }\end{array}$ \\
\hline Subjects $n$ & 16 & 12 & 14 & & & & & & \\
\hline BV1 $\mathbf{n g} \cdot \mathrm{mL}^{-1}$ & $84.8(46.8-120.0)$ & $78.8(40.8-125.2)$ & $110.4(70.2-196.6)$ & 0.001 & 0.610 & 0.001 & 0.001 & $<0.001$ & 0.090 \\
\hline BV2 $\mathbf{n g} \cdot \mathrm{mL}^{-1}$ & $72.6(32.1-103.5)$ & $83.6(45.4-112.0)$ & $126.7(76.8-173.2)$ & $<0.001$ & 0.194 & $<0.001$ & $<0.001$ & $<0.001$ & 0.001 \\
\hline BV3 $\mathbf{n g} \cdot \mathrm{mL}^{-1}$ & $51.7(25.5-90.2)$ & $101.1(55.3-138.1)$ & $159.7(111.7-233.4)$ & $<0.001$ & $<0.001$ & $<0.001$ & 0.001 & $<0.001$ & $<0.001$ \\
\hline BV1-BV2 \% decrease & $11.8(-34.4-62.8)$ & $0.7(-41.8-32.1)$ & $-13.8(-30.1-20.5)$ & 0.042 & 0.095 & 0.015 & 0.572 & 0.064 & 0.014 \\
\hline
\end{tabular}

Data are presented as median (range) unless otherwise indicated. BV1: baseline cfDNA level before first chemotherapy cycle; R: remission; SD: stable disease; P: progression. \#: R versus SD versus $\mathrm{P}$ (Kruskal-Wallis test); $"$ : Wilcoxon rank-sum test (Bonferroni correction applied; $p<0.017$ was taken as significant) indicates differences between the compared groups; ${ }^{+}$: Wilcoxon rank-sum test indicates differences between patients in the evaluations shown. Significant observations are shown in bold.

correlation between other prognostic factors and survival was observed (table 1).

\section{Evaluation of cfDNA levels in various response groups}

The BV1, BV2 and BV3 were quantified in 42 patients with advanced-stage NSCLC. Overall, a significant difference in cfDNA levels was observed among all three groups (R, SD and $\mathrm{P})$. When compared among any two groups, significantly lower BV1, BV2 and BV3 were observed in patients with R or $\mathrm{SD}$ as compared to patients with $\mathrm{P}$ (table 2 ). However, patients with $\mathrm{R}$ were distinguished from patients with SD only by the BV3 (table 2). cfDNA levels decreased in patients with $\mathrm{R}$ but increased in patients with $\mathrm{SD}$ or $\mathrm{P}$ during chemotherapy (table 2; fig. 1). The fraction of patients with lower BV2 than BV1 in the various groups were $75.0(\mathrm{R}), 50.0$ (NC) and $35.7 \%$

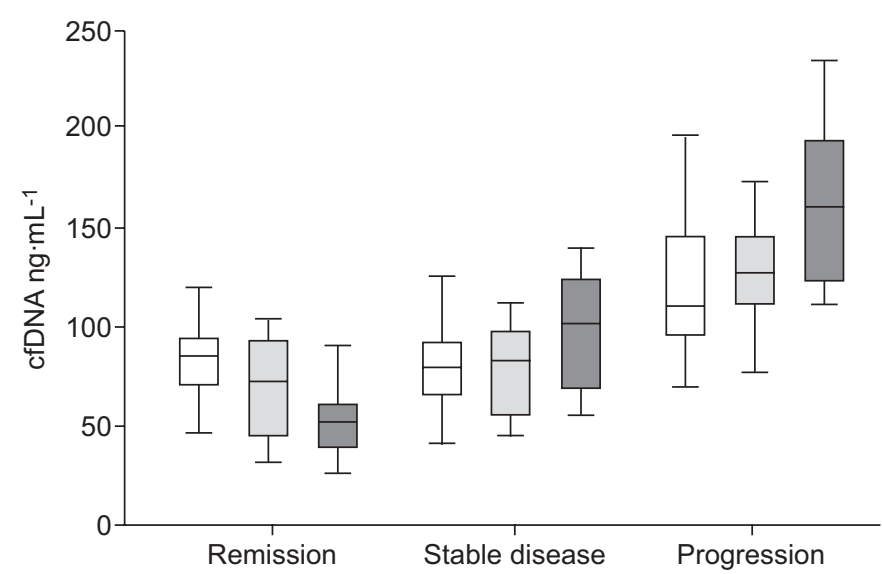

FIGURE 1. Distribution of cell-free plasma DNA (cfDNA) levels in various response groups before the start of the first $(\square)$, second $(\square)$ and third $(\square)$ chemotherapy cycles. Boxes represent median and interquartile range; vertical bars show range.
$(\mathrm{P})$, and with lower BV3 than BV1 were $100(\mathrm{R}), 25(\mathrm{NC})$ and $0.0 \%(\mathrm{P})$, respectively.

The kinetics of cfDNA from cycle 1 to 2 , cycle 1 to 3 and cycle 2 to 3 were also assessed in correlation with its efficacy in distinguishing various response groups (table 2). A significant decrease in cfDNA levels was observed from cycle 1 to 3 and from cycle 2 to 3 in patients with $R$ as compared to those with $P$ (table 2). The kinetics of cfDNA from cycle 1 to 3 and cycle 2 to 3 was able to distinguish patients with $\mathrm{R}$ from those with SD. However, the kinetics of cfDNA was not able to distinguish patients with SD from those with $\mathrm{P}$ (table 2).

\section{Evaluation of cfDNA levels for predicting $P$ and insufficient response to therapy}

In order to assess the efficacy of cfDNA levels in predicting insufficient response to therapy and $P$ in patients with NSCLC, BV1, BV2 and BV3 were compared in different response groups. Although pretreatment cfDNA levels (BV1) could not predict insufficient response to therapy $(p<0.09)$, they were able to predict $\mathrm{P}(\mathrm{p}<0.001$; table 2$)$. Further, BV2 and BV3 were able to predict insufficient response to therapy and $P$. Additionally, the kinetics of cfDNA levels from cycle 1 to 2 (BV1-BV2), cycle 1 to 3 (BV1-BV3) and cycle 2 to 3 (BV2-BV3) was able to predict insufficient response to therapy (table 2).

\section{Diagnostic profile of cfDNA levels during chemotherapy for predicting insufficient response to therapy and $P$}

In order to test the potential of cfDNA levels as a biomarker for the prediction of insufficient response to therapy, ROC curves were plotted for BV1, BV2 and BV3. Using BV1, insufficient response to therapy could be predicted with a sensitivity of $26.9 \%$ at absolute $(100 \%)$ specificity (AUC 0.657 ; cut-off value $\left.121.1 \mathrm{ng} \cdot \mathrm{mL}^{-1}\right)$. Further, BV2 and BV3 were able to predict insufficient response to therapy with a sensitivity of $50.0 \%$ (AUC 0.805; cut-off value $105.8 \mathrm{ng} \cdot \mathrm{mL}^{-1}$ ) and $80.8 \%$ (AUC 
0.954; cut-off value $93.8 \mathrm{ng} \cdot \mathrm{mL}^{-1}$ ), respectively, at $100 \%$ specificity.

The relevance of BV1, BV2 and BV3 in predicting $\mathrm{P}$ was also assessed (fig. 2). From the ROC curve, it was observed that, among all of the values, BV2 most efficiently identified patients with $\mathrm{P}$ with a sensitivity of $71.4 \%$ at $100 \%$ specificity (AUC 0.926; cut-off value $115.6 \mathrm{ng} \cdot \mathrm{mL}^{-1}$ ). Further, BV1 and BV3 were able to predict $\mathrm{P}$ with a sensitivity of $35.7 \%$ (AUC 0.853 ; cut-off value $132.5 \mathrm{ng} \cdot \mathrm{mL}^{-1}$ ) and $64.3 \%$ (AUC 0.952 ; cut-off value $\left.140.6 \mathrm{ng} \cdot \mathrm{mL}^{-1}\right)$, respectively, at $100 \%$ specificity.

\section{cfDNA levels as a prognostic marker in NSCLC}

The correlation between cfDNA levels before various treatment cycles and survival time was investigated in 42 NSCLC patients who received chemotherapy and for whom survival data were available. With the median cfDNA level as the cut-off value, patients were divided into high- (above or equal to median) and low (below median)-cfDNA groups. No correlation was observed between BV1 and BV2 and survival (table 3). However, BV3 was significantly related to survival (table 3).

\section{DISCUSSION}

The presence of circulating tumour DNA in the serum/plasma of cancer patients has sparked great interest because conventional diagnostic tests tend to be imperfect and more invasive, posing logistic difficulties for serial tumour sampling. Lessinvasive techniques, such as blood tests, are attractive for screening, diagnosis, prognosis, surveillance for occult $\mathrm{P}$, identification of potential therapeutic targets and monitoring of tumour responses. With the advance in laboratory techniques, it has now become possible to isolate and quantify cfDNA from easily accessible plasma/serum and other body fluids, such as lavage fluids (obtained from various organs), urine and ascites [12]. Along with the finding that cfDNA originates from tumours [12], such analysis may reflect all of the physiological and pathological processes going on in the human body, thus making them a very interesting target for the development of useful clinical assays. Further, such analysis may be commercially viable owing to their easy

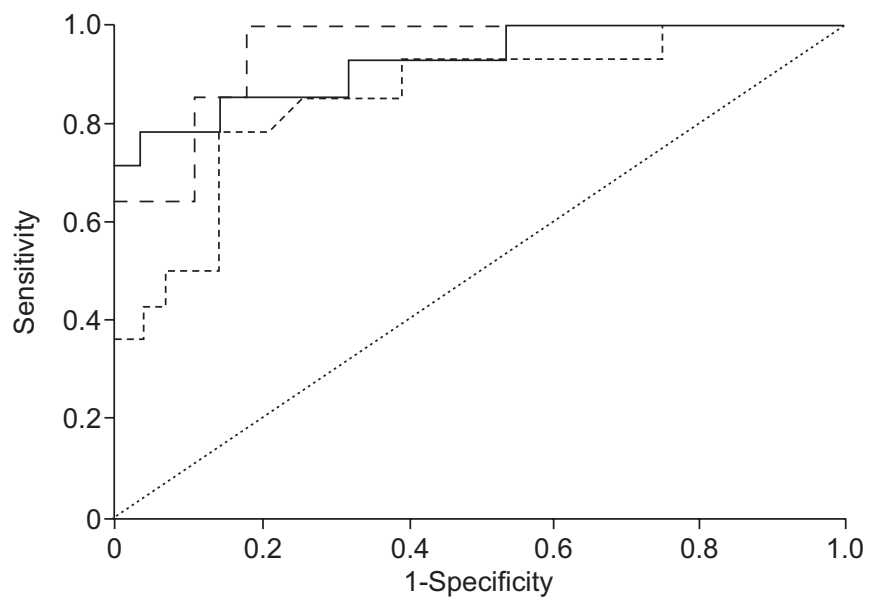

FIGURE 2. Receiver operating characteristic curves for predicting disease progression by cell-free plasma DNA levels before the start of the first (--------; area

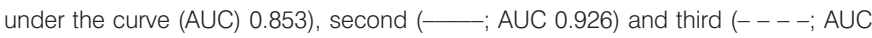
$0.952)$ cycle of chemotherapy ( $\cdots \cdots \cdots \cdot$ : reference line)

\begin{tabular}{|c|c|c|c|}
\hline TABLE 3 & \multicolumn{3}{|c|}{$\begin{array}{l}\text { Correlation between cell-free plasma DNA } \\
\text { (cfDNA) levels and survival in nonsmall cell lung } \\
\text { cancer }\end{array}$} \\
\hline \multicolumn{2}{|c|}{ Cut-off value $\mathrm{ng} \cdot \mathrm{mL}^{-1}$} & Survival days & $p$-value \\
\hline \multicolumn{4}{|l|}{ BV1 } \\
\hline$\geqslant 90.2$ & & $328.0(314.8-341.2)$ & 0.342 \\
\hline$<90.2$ & & $403.0(339.0-467.0)$ & \\
\hline \multicolumn{4}{|l|}{ BV2 } \\
\hline$\geqslant 93.8$ & & $327.0(312.9-341.1)$ & 0.235 \\
\hline$<93.8$ & & $416.0(329.5-502.5)$ & \\
\hline \multicolumn{4}{|l|}{ BV3 } \\
\hline$\geqslant 93.8$ & & $324.0(303.6-344.4)$ & $<0.001$ \\
\hline$<93.8$ & & 462.0 (328.4-595.6) & \\
\hline
\end{tabular}

Data are presented as median or median (95\% confidence interval). BV1 baseline cfDNA level before first chemotherapy cycle

availability in the market and their cost-effectiveness comparing to more expensive imaging tools.

The first evidence supporting the tumoural origin of plasma DNA in cancer patients was provided in the late 1980s [13]. However, the final proof that tumours can shed DNA into the circulation came from two studies that detected oncogenic $\mathrm{N}$ ras mutations in the plasma of patients with myelodysplastic syndrome and acute myelogenous leukaemia [14], and K-ras mutations in the plasma and serum of patients with pancreatic adenocarcinoma [15], respectively. Since then, numerous groups have studied the quantitative and qualitative aspects of circulating DNA in cancer, although the sensitivity and specificity of the various methods utilised have shown great variability $[8,12]$. Significantly higher plasma DNA levels were found in NSCLC patients than in controls with various benign pulmonary diseases. This suggests that the increased presence of cfDNA in the plasma of lung cancer patients might be due to apoptosis, necrosis, tumour cell lysis or the excessive turnover rate of tumour cells. Further, findings in mice bearing human tumours and undergoing chemotherapy or surgery suggested that cfDNA can be used for monitoring the effect of therapy $[16,17]$. In mice bearing human tumours, a marked transient rise in the levels of human-specific cfDNA (tumour-specific DNA) occurred immediately after chemotherapy or surgery, followed by a rapid decrease [16, 17]. These observations indicated that the kinetics of tumour-specific DNA in plasma may reflect tumour burden, i.e. the rapid cell death following treatment may release tumour cell DNA into the circulation, which decreases as the tumour regresses. These studies indicate that analysis of cfDNA may play a potential role in monitoring the efficacy of cancer therapies.

In the present study, the trend in cfDNA levels analysed at three different time-points in advanced-stage NSCLC patients receiving first-line platinum-based chemotherapy was correlated with response to therapy. Comparable BV1 and BV2 were found in patients with R and SD. However, when compared with patients with $\mathrm{P}$, patients with $\mathrm{R}$ or $\mathrm{SD}$ showed significantly lower BV1-BV3. Similar results were also reported for serum nucleosome levels (cfDNA is associated 
with histone proteins) measured before various cycles of chemotherapy in patients with NSCLC [18] and small cell lung cancer (SCLC) [19]. During the course of chemotherapy, a decrease in cfDNA levels was observed in patients with $\mathrm{R}$, whereas the levels increased in patients with SD or P. There is a paucity of published work on the utility of cfDNA as a monitoring tool for lung cancer receiving chemotherapy. In a previous study, a significant decrease in serum DNA levels was observed in patients with $\mathrm{SD}$ and R; however, plasma DNA concentrations remained unchanged [20]. In the same study, patients with $\mathrm{P}$ showed a significant increase in plasma DNA concentrations, an observation similar to the present one. A decline in post-treatment plasma DNA levels has also been observed previously [21]. Plasma DNA concentrations have also been observed to decline significantly in lung cancer patients after undergoing surgery [22]. In a study on rectal cancer patients undergoing chemoradiation, median plasma DNA levels before and after the end of treatment were comparable in responders and nonresponders [23]. However, at the end of treatment, responders showed a further decrease in circulating DNA level, whereas, in nonresponders, the circulating DNA manifestly increased. These findings suggest that monitoring the levels of cfDNA during the course of cytotoxic therapies may help in predicting response to therapy. It is necessary to mention here that, as the present study dealt with only a limited number of NSCLC patients, validation of these comparisons for differentiation of various response groups needs to be performed on a larger sample size.

The present results with cfDNA are in accordance with the hypothesis that, in responders, any decrease in tumour mass due to cytotoxic therapies causes a substantial decrease in the levels of tumour markers. Conversely, there is either a mild decrease or an increase in marker levels in nonresponders due to insufficient therapy response. It should be noted that there are considerable numbers of dying cells during chemotherapy, thus releasing cfDNA into the circulation of both responders and nonresponders. The significant difference observed in cfDNA levels between responders and nonresponders may not only reflect the change in tumour load due to cytotoxic therapies but also factors that may also influence the levels of cfDNA, such as: 1) activity of DNases [24], 2) efficiency of cfDNA clearance from the circulation [25, 26], 3) macrophage number and function [27], 4) activity, proliferation and turnover rate of the residual tumour mass, 5) dysfunctional DNA repair mechanisms, and 6) the blood supply of tumour tissues that facilitate the transition of cfDNA into the blood circulation.

A significant difference in overall survival time was observed between patients who went into $\mathrm{R}$ as compared to patients with $\mathrm{SD}$ or P. As patients with no change in disease status, i.e. $\mathrm{SD}$, they are those in whom tumour reduction was insufficient to be classified as $\mathrm{R}$ or an increase in tumour growth was insufficient to be considered as P. Thus it would be useful to find patients who are going to either: 1) respond to the treatment, or 2) progress to a more advanced stage of disease at an early stage. For both of these groups, it would be necessary to focus on the biomarkers already available before the start of the first or second course of treatment. This could be helpful in the better management of these patients since prediction of insufficient response to therapy or $\mathrm{P}$ at an early stage can be used to either intensify treatment or change the treatment plan. Therefore, two different evaluations of cfDNA levels before various treatment cycles were conducted in order to address both clinical questions.

For predicting $\mathrm{P}$ (evaluation $1 ; \mathrm{R}+\mathrm{SD}$ versus $\mathrm{P}$ ), it was observed that pretreatment cfDNA levels were able to predict $\mathrm{P}$, an observation that contrasts with a previous study on SCLC [19]. Further, BV2 predicted P with the greatest sensitivity $(71.4 \%)$ at $100 \%$ specificity. This is much higher than the sensitivity of $29 \%$ at $100 \%$ specificity using serum nucleosomes measured on day 8 of the first chemotherapy cycle and CYFRA 21-1 (cytokeratin 19 fragments) measured before cycle 2 [18]. It is to be noted that, since the specificity is absolute, none of the responders are harmed by modifying the treatment plan at an early stage using this information. In a previous study on SCLC patients, a sensitivity of 30 and $40 \%$ was achieved at $95 \%$ specificity for predicting $\mathrm{P}$ using serum nucleosome levels before the second and third cycle of chemotherapy, respectively [19].

For predicting insufficient response to therapy (evaluation 2; $\mathrm{R}$ versus $\mathrm{SD}+\mathrm{P}$ ), it was observed that, except for BV1, cfDNA levels before various chemotherapy cycles were able to predict insufficient response to therapy, an observation also reported previously in SCLC [19]. It should be noted that, for predicting insufficient treatment efficacy, biomarkers should not misclassify responders as nonresponders since this would lead to cessation of successful therapy. Taking this into account, it was possible to predict insufficient response to therapy with a sensitivity of 26.9 and $50.0 \%$ at $100 \%$ specificity using BV1 and BV2, respectively. This again, scores better than the sensitivity achieved by measuring serum nucleosomes before the second cycle of chemotherapy in NSCLC (31.4\% at 90\% specificity) [18], as well as SCLC (23.5\% at 95\% specificity) patients [19].

The correlation between plasma levels of cfDNA measured before various treatment cycles and survival time was also analysed in an effort to analyse its utility as a prognostic marker for survival in NSCLC patients. A correlation was observed between BV3 and survival; i.e. patients with lower cfDNA levels survived longer. The existing data regarding the prognostic relevance of pretreatment cfDNA level in lung cancer are scant and conflicting [20, 22, 28-31]. Some studies have demonstrated correlation between an elevated plasma DNA concentration and poor survival [20, 28, 30, 31], whereas others, including the present work, have not [22, 29]. Possible explanations for the discrepancy in survival data may be variation in patient selection, combining NSCLC and SCLC together [28, 29], and/or different methods of DNA isolation and quantification. Since NSCLC and SCLC differ in biology, treatment and prognosis, only NSCLC patients were included in the present study.

One of the limitations of the present study was that plasma DNA levels were quantified using the fluorescence-based PicoGreen method. For DNA quantification, the quantitative real-time PCR (qPCR) method has been regarded as the gold standard since it can detect a large range of small amounts of nucleic acids. Also, qPCR is characterised by high accuracy and high sensitivity. However, it requires specific automated systems and remains expensive, thus limiting its commercial applicability. Further, the use of various gene-specific primers 
or probe sequences and availability of numerous real-time PCR devices requires the optimisation of qPCR reaction conditions, which hampers the comparison of data from different laboratories [32]. Other techniques, such as radioimmunoassay and the common bench ultraviolet-spectrophotometer method cannot detect DNA concentrations below nanogram levels. The PicoGreen method demonstrates an exceptionally high detection limit, up to $25 \mathrm{pg} \cdot \mathrm{mL}^{-1}$, and perfect linearity up to $1,000 \mathrm{ng} \cdot \mathrm{mL}^{-1}$, making the test appropriate for circulating DNA analysis. Additionally, a strong and positive correlation has been observed between the PicoGreen assay and qPCR [33]. Further, a simple uniform protocol and microplate assay format makes the PicoGreen assay a rapid, effective and inexpensive tool for high-throughput quantification of cfDNA, especially for serial assessment for screening, diagnostic or prognostic purposes.

A recent study has shown the effect of storage time of whole plasma and purified DNA on the levels of plasma DNA quantified from lung cancer patients and age-matched controls using a real-time PCR-based assay [34]. In that study, plasma DNA levels declined at a mean rate of $\sim 30 \%$ annually when two samples of plasma and isolated DNA were assessed for each patient with a median gap of 41 months between the two assessments. The authors concluded that care must be taken while performing large-scale prospective studies or retrospective studies with stored plasma samples or purified DNA. In the present study, the time from plasma separation and storage to performance of plasma DNA extraction and quantification was $<6$ months in order to avoid any such time-dependent variation. In addition, plasma samples were analysed with a comparable storage time and storage temperature in order to ensure that issues relating to timedependent degradation of cfDNA could be avoided [34]. It is evident that any future application of such analysis will depend upon the reproducibility and reliability of results, both of which require the optimisation and equivalence of various pre-analytical and analytical factors.

In conclusion, small sample size was a limitation of the present study; however, we feel that the results are promising enough to encourage further research into this aspect of cancer therapeutics. The present study shows that monitoring of cfDNA levels during the course of cytotoxic therapy can predict an early response to therapy and $P$. There is only limited information available regarding the predictive value of cfDNA in NSCLC patients. Thus more external, multicentric and large-scale prospective validation studies are required in order to confirm the relevance of the current findings for the early estimation of response to therapy and prognosis. Additionally, identification of additional more-specific and more-sensitive serum- or plasma-based biomarkers, which can be used in combination with cfDNA, will further improve the diagnostic power of current imaging tools for indicating early therapeutic efficacy. Such analysis may also have potential in monitoring tumour recurrence and as intermediate end-points of efficacy in chemoprevention and therapeutic trials. Such analysis may also be used in present clinical practice, where efforts are made to treat patients based on their epidermal growth factor receptor mutation status [35] or during secondand third-line treatment in advanced NSCLC with the drugs doxetaxel, pemetrexed or erlotinib [36]. It will be interesting to see how cfDNA performs in this new therapeutic setting.

\section{SUPPORT STATEMENT}

This study was funded by the Indian Council for Medical Research (New Delhi, India). S. Kumar is in receipt of a Council of Scientific and Industrial Research (New Delhi, India) Senior Research Fellowship.

\section{STATEMENT OF INTEREST}

None declared.

\section{REFERENCES}

1 Parkin DM, Bray F, Ferlay J, et al. Global cancer statistics, 2002. CA Cancer J Clin 2005; 55: 74-108.

2 Gridelli C, Rossi A, Maione P. Treatment of non-small cell lung cancer: state of the art and development of new biological agents. Oncogene 2003; 22: 6629-6638.

3 Pfister DG, Johnson DH, Azzoli CG, et al. American Society of Clinical Oncology treatment of unresectable non-small cell lung cancer guideline: update 2003. J Clin Oncol 2004; 22: 330-353.

4 Spira A, Ettinger DS. Multidisciplinary management of lung cancer. N Engl J Med 2004; 350: 379-392.

5 Weber WA, Petersen V, Schmidt B, et al. Positron emission tomography in non-small-cell lung cancer: prediction of response to chemotherapy by quantitative assessment of glucose use. J Clin Oncol 2003; 21: 2651-2657.

6 Pottgen C, Levegrun S, Theegarten D, et al. Value of ${ }^{18} \mathrm{~F}$-fluoro-2deoxy-D-glucose-positron emission tomography/computed tomography in non-small-cell lung cancer for prediction of pathologic response and times to relapse after neoadjuvant chemoradiotherapy. Clin Cancer Res 2006; 12: 97-106.

7 de Geus-Oei LF, van der Heijden HF, Corstens FH, et al. Predictive and prognostic value of FDG-PET in non-small cell lung cancer: a systematic review. Cancer 2007; 110: 1654-1664.

8 Pathak AK, Bhutani M, Kumar S, et al. Circulating cell-free DNA in plasma/serum of lung cancer patients as a potential screening and prognostic tool. Clin Chem 2006; 52: 1833-1842.

9 Mountain CF. Revision in the international system for staging lung cancer. Chest 1997; 111: 1710-1717.

10 Gajalakshmi V, Hung RJ, Mathew A, et al. Tobacco smoking and chewing, alcohol drinking and lung cancer risk among men in Southern India. Int J Cancer 2003; 107: 441-447.

11 Miller $A B$, Hoogstraten $B$, Staquet $M$, et al. Reporting results in cancer treatment. Cancer 1981; 47: 207-214.

12 Fleischhacker M, Schmidt B. Circulating nucleic acids (CNAs) and cancer - a survey. Biochim Biophys Acta 2007; 1775: 181-232.

13 Stroun M, Anker P, Maurice P, et al. Neoplastic characteristics of the DNA found in the plasma of cancer patients. Oncology 1989; 46: 318-322.

14 Vasioukhin V, Anker P, Maurice P, et al. Point mutations of the Nras gene in the blood plasma of patients with myelodysplastic syndrome or acute myelogenous leukaemia. Br J Haematol 1994; 86: 774-779.

15 Sorenson GD, Pribish DM, Valone FH, et al. Soluble normal and mutated DNA sequences from single-copy genes in human blood. Cancer Epidemiol Biomarkers Prev 1994; 3: 67-71.

16 Kamat AA, Bischoff FZ, Dang D, et al. Circulating cell-free DNA: a novel biomarker for response to therapy in ovarian carcinoma. Cancer Biol Ther 2006; 5: 1369-1374.

17 Rago C, Huso DL, Diehl F, et al. Serial assessment of human tumor burdens in mice by the analysis of circulating DNA. Cancer Res 2007; 67: 9364-9370.

18 Holdenrieder S, Stieber P, von Pawel J, et al. Early and specific prediction of the therapeutic efficacy in lung cancer by nucleosomal 
DNA and cytokeratin-19 fragments. Ann N Y Acad Sci 2006; 1075: 244-257.

19 Holdenrieder S, von Pawel J, Dankelmann E, et al. Nucleosomes, ProGRP, NSE, CYFRA 21-1, and CEA in the therapy monitoring of small-cell lung cancer during first-line chemotherapy. Clin Cancer Res 2008; 14: 7813-7821.

20 Gautschi O, Bigosch C, Huegli B, et al. Circulating deoxyribonucleic acid as a prognostic marker in non-small cell lung cancer patients undergoing chemotherapy. J Clin Oncol 2004; 22: 4157-4164.

21 Sozzi G, Conte D, Leon M, et al. Quantification of free circulating DNA as a diagnostic marker in lung cancer. J Clin Oncol 2003; 21: 3902-3908.

22 Sozzi G, Conte D, Mariani L, et al. Analysis of circulating tumor DNA in plasma at diagnosis and during follow-up of lung cancer patients. Cancer Res 2001; 61: 4675-4678.

23 Zitt M, Muller HM, Rochel M, et al. Circulating cell-free DNA in plasma of locally advanced rectal cancer patients undergoing preoperative chemoradiation: a potential diagnostic tool for therapy monitoring. Dis Markers 2008; 25: 159-165.

24 Economidou-Karaoglou A, Brasseur F, Lans M, et al. Variations in serum alkaline DNase activity in rats during growth and treatment of tumors sensitive or resistant to therapy. Int J Cancer 1989; 43: 956-959.

25 Burlingame RW, Volzer MA, Harris J, et al. The effect of acute phase proteins on clearance of chromatin from the circulation of normal mice. J Immunol 1996; 156: 4783-4788.

26 Gauthier VI, Tyler LN, Mannik M. Blood clearance kinetics and liver uptake of mononucleosomes in mice. J Immunol 1996; 156: 1151-1156.

27 Odaka C, Mizuochi T. Role of macrophage lysosomal enzymes in the degradation of nucleosomes of apoptotic cells. J Immunol 1999; 163: 5346-5352.
28 Fournie GJ, Courtin JP, Laval F. Plasma DNA as a marker of cancerous cell death: investigation in patients suffering from lung cancer and in nude mice bearing human tumour. Cancer Lett 1995; 2: 221-227.

29 Beau-Faller M, Gaub MP, Schneider A, et al. Plasma DNA microsatellite panel as sensitive and tumor-specific marker in lung cancer patients. Int J Cancer 2003; 105: 361-370.

30 Sozzi G, Roz L, Conte D, et al. Plasma DNA quantification in lung cancer computed tomography screening. Am J Respir Crit Care Med 2009; 179: 69-74.

31 van der Drift MA, Hol BE, Klaassen $\mathrm{CH}$, et al. Circulating DNA is a non-invasive prognostic factor for survival in non-small cell lung cancer. Lung Cancer 2010; 68: 283-287.

32 Chorostowska-Wynimko J, Szpechcinski A. The impact of genetic markers on the diagnosis of lung cancer: a current perspective. J Thorac Oncol 2007; 2: 1044-1051.

33 Szpechcinski A, Struniawska R, Zaleska J, et al. Evaluation of fluorescence-based methods for total vs. amplifiable DNA quantification in plasma of lung cancer patients. J Physiol Pharmacol 2008; 59: Suppl. 6, 675-681.

34 Sozzi G, Roz L, Conte D, et al. Effects of prolonged storage of whole plasma or isolated plasma DNA on the results of circulating DNA quantification assays. J Natl Cancer Inst 2005; 97: $1848-1850$.

35 Gazdar AF. Personalized medicine and inhibition of EGFR signaling in lung cancer. N Engl J Med 2009; 361: 1018-1020.

36 de Marinis F, Grossi F. Clinical evidence for second- and third-line treatment options in advanced non-small cell lung cancer. Oncologist 2008; 13: 14-20. 